\title{
Comparing Prostate Imaging-Reporting and Data System Version 2 (PI-RADSv2) Category 1 and 2 Groups: Clinical Implication of Negative Multiparametric Magnetic Resonance Imaging
}

\author{
Jung Kwon Kim, ${ }^{1}$ Hak Jong Lee, ${ }^{2}$ Sung Il Hwang, ${ }^{2}$ Gheeyoung Choe, ${ }^{3}$ \\ and Sung Kyu Hong $\mathbb{D}^{1,4}$ \\ ${ }^{1}$ Department of Urology, Seoul National University Bundang Hospital, Seongnam, Republic of Korea \\ ${ }^{2}$ Department of Radiology, Seoul National University Bundang Hospital, Seongnam, Republic of Korea \\ ${ }^{3}$ Department of Pathology, Seoul National University Bundang Hospital, Seongnam, Republic of Korea \\ ${ }^{4}$ Department of Urology, Seoul National University College of Medicine, Seoul, Republic of Korea
}

Correspondence should be addressed to Sung Kyu Hong; skhong@snubh.org

Received 16 September 2019; Accepted 24 March 2020; Published 2 April 2020

Academic Editor: Pradeep Tyagi

Copyright (C) 2020 Jung Kwon Kim et al. This is an open access article distributed under the Creative Commons Attribution License, which permits unrestricted use, distribution, and reproduction in any medium, provided the original work is properly cited.

\begin{abstract}
Objectives. To evaluate the clinicopathological differences between Prostate Imaging-Reporting and Data System (PI-RADS) version 2 (v2) category 1 and 2 groups. Materials and Methods. We retrospectively reviewed our two institutional clinical databases: (1) transrectal ultrasound (TRUS)/magnetic resonance imaging (MRI) fusion biopsy cohort $(n=706)$ and (2) radical prostatectomy (RP) cohort $(n=1403)$. Subsequently, we performed comparative analyses between PI-RADSv2 category 1 and 2 groups. Clinically significant prostate cancer (csPCa) was defined as the presence of Gleason score (GS) $\geq 3+4$ in a single biopsy core, and adverse pathology (AP) was defined as high-grade (primary Gleason pattern 4 or any pattern 5) and/or nonorgan-confined disease (pT3/N1). We also performed multivariate logistic regression analyses for AP. Results. In the TRUS/MRI fusion biopsy cohort, no significant differences in detection rates of all cancer (18.2\% vs. $29.0 \%$, respectively, $P=0.730)$ or csPCa (9.1\% vs. 9.9\%, respectively, $P=0.692)$ were observed between PI-RADSv2 category 1 and 2 groups. There were no significant differences in pathologic outcomes including Gleason score $(\geq 4+3,21.2 \%$ vs. $29.9 \%$, respectively, $P=0.420)$ or detection rate of AP $(27.3 \%$ vs. $33.8 \%$, respectively, $P=0.561)$ between the two groups in the RP cohort either. PI-RADSv2 category 1 or 2 had no significant association with AP, even in univariate analysis $(P=0.299)$. Conclusions. PI-RADSv2 categories 1 and 2 had similar performance to predict clinicopathological outcomes. Consequently, these two categories may be unified into a single category. Negative mpMRI does not guarantee the absence of AP, as with csPCa.
\end{abstract}

\section{Introduction}

In the past decade, there has been growing body of evidence that suggests a role of multiparametric magnetic resonance imaging (mpMRI) in the detection and management of prostate cancer (PCa). The latest European Association of Urology (EAU) guidelines on PCa strongly recommend mpMRI before repeat biopsy when clinical suspicion of PCa persists despite previous negative biopsies [1]. In 2016, the European Society of Urogenital Radiology (ESUR) released an updated guideline termed Prostate Imaging Reporting and Data System version 2 (PI-RADSv2) to ensure standardized and reliable radiologic criteria $[2,3]$. In a previous meta-analysis, Woo et al. [4] have reported that PI-RADSv2 has high pooled sensitivity of $89 \%$ and specificity of $73 \%$. However, they also demonstrated that there was significant variability in published results. Negative predictive value (NPV) to exclude clinically significant $\mathrm{PCa}$ (csPCa) was highly variable, ranging from $63 \%$ to $98 \%$ [5]. These variable results could be explained by differences in patient populations, reference standards, image acquisition techniques, image quality, interpretation criteria, reader experience, and interreader variability $[4,6]$.

Notably, in the majority of studies using PI-RADS, a category of $\geq 3$ was considered as targetable lesion for biopsy to 
evaluate its performance in detecting PCa [1, 7-15]. Studies on PI-RADS category 1 and 2 lesions are lacking [11-14]. To the best of our knowledge, no studies have directly compared category 1 and 2 lesions. To further improve strategies, larger and longer-term follow-up data of these "negative" mpMRI patients would be essential. Accurate preoperative PCa prediction is essential for patient counseling as well as treatment approach. Accordingly, mpMRI plays an important role in predicting the final pathology at radical prostatectomy (RP). However, significant rate of mpMRI lesions does not always correspond to guided biopsy or RP specimen findings [16]. In addition, concordance rate between biopsy and RP Gleason score (GS) is only $35 \%$ to as high as $90 \%[17,18]$. Regarding this disconcordance, previous studies have demonstrated the association of PI-RADS with upgrading and extraprostatic extension (EPE) in RP pathology [19-21]. Thus, the aim of the present study was to evaluate pathologic outcomes after both biopsy and RP in patients with "negative" (PI-RADSv2 category $\leq 2$ ) mpMRI. We also evaluated clinicopathological differences between PI-RADSv2 category 1 and 2 groups.

\section{Methods}

2.1. Ethics Statement. The Institutional Review Boards of the Seoul National University Bundang Hospital approved this study (Approval number: B-1706/402-115). As the present study was carried out retrospectively, written informed consent from patients was waived. Personal identifiers were completely removed and the data were analyzed anonymously. Our study was conducted according to the ethical standards recommended by the 1964 Declaration of Helsinki and its later amendments.

\subsection{Study Cohort}

\subsubsection{Transrectal Ultrasound (TRUS)/MRI Fusion Biopsy} Cohort. We reviewed our institutional TRUS/MRI fusion biopsy database between September 2015 and March 2018 retrospectively. Accordingly, a total of 706 patients were included separately to the RP cohort.

2.2.2. Radical Prostatectomy Cohort. We also reviewed clinical data of patients who underwent RP and preoperative 3-tesla (3 T) multiparametric prostate MRI (mpMRI) for clinically localized or locally advanced PCa between March 2008 and April 2018 at our institution retrospectively. A total of 1403 patients were finally included in this study after excluding 25 patients who underwent neoadjuvant treatment.

2.3. Preoperative MRI Protocol and Image Interpretation. All preoperative mpMRIs were performed after biopsy (usually 2 to 6 weeks later) using a $3 \mathrm{~T}$ system (Achieva Tx and Ingenia; Philips, the Netherlands) with a phase-array cardiac 6-channel coil without using the endorectal coil. mpMRI consisted of axial T2-weighted imaging (T2WI), T1/T2-weighted registered imaging (T1/T2RI), diffusionweighted imaging (DWI) with corresponding apparentdiffusion coefficient (ADC) maps, and dynamic contrast enhanced (DCE). Detailed protocols were described in our previous reports [22-24]. All images were reviewed by two high-volume radiologists (H.J.L. and S.I.H.) who had $>20$ years of experience in interpreting prostate MRI using a Picture Archiving and Communication Systems workstation (PACS, INFINITT Technology, Seoul, Korea). mpMRI lesions were categorized through PI-RADSv2 [3]. Subsequently, all images from 2008 to early 2016 were rereviewed according to PI-RADSv2. Two radiologists were blinded to clinical characteristics including pathologic outcomes at the time of interpretation. However, they were aware that these patients had pathologically confirmed PCa.

2.4. TRUS/MRI Fusion Biopsy Protocol. From September 2015, two high-volume radiologists (S.I.H. and H.J.L.) in our institution started to conduct TRUS/MRI fusion biopsy [24]. $3 \mathrm{~T}$ mpMRI was performed before the biopsy in all patients. All mpMRI lesions were scored through PI-RADSv2, and images before early 2016 were also rereviewed according to PI-RADSv2. The fusion imaging technique (Volume Navigation; GE Healthcare, USA) with an electromagnetic field tracking system was used. Before the study, the axial MR images were uploaded from the PACS archive to the TRUS machine. After that, registration between the TRUS and MR images was performed to fuse both images correctly. In case of two index lesions in the same patient, the registrations were performed again for the subsequent lesion after the first biopsy. All TRUS-guided biopsies were performed with a Logiq E9 US machine (GE Healthcare, USA) equipped with a 5-9 MHz multifrequency endocavitary probe by the same uroradiologist who had conducted the image fusion [24]. An 18-gauge, $20 \mathrm{~cm}$ automatic cutting needle and an automated biopsy gun (ACECUT, TSK Laboratory, Japan) were used [24, 25].

Patients with index lesions of PI-RADSv2 category $\geq 3$ were classified as the MRI-positive group, while patients with PI-RADSv2 category $\leq 2$ as the MRI-negative group. Two cores of additional biopsy were performed per each index lesion under TRUS/MRI fusion. The maximal number of additional biopsy was four in a patient. Twelve cores of randomized systematic biopsy were followed. In the MRI-negative group, two cores of additional biopsy were conducted in the transition zone, followed by systematic biopsy. csPCa was defined as the presence of Gleason score $(\mathrm{GS}) \geq 3+4$ in a single biopsy core [8].

2.5. Data Acquisition and Definitions. RPs were conducted by several surgeons (S.E.L., S.S.B., S.L., and S.K.H.) using open, laparoscopic, or robotic modality. All pathological specimens were evaluated by a staff pathologist (G.C.) who had genitourinary expertise. Adverse pathology (AP) was defined as high-grade (primary Gleason pattern 4 or any pattern 5 ) and/or non-organ-confined disease (pT3/N1) [26]. The following variables were compared between categorical groups: age, body mass index (BMI), prebiopsy prostate-specific antigen (PSA) level, biopsy and pathologic GS, prostate volume on TRUS, PSA density (PSAD, serum PSA level/prostate volume) [27], and National Comprehensive Cancer Network (NCCN) criteria for prostate cancer risk assessment [28]. 


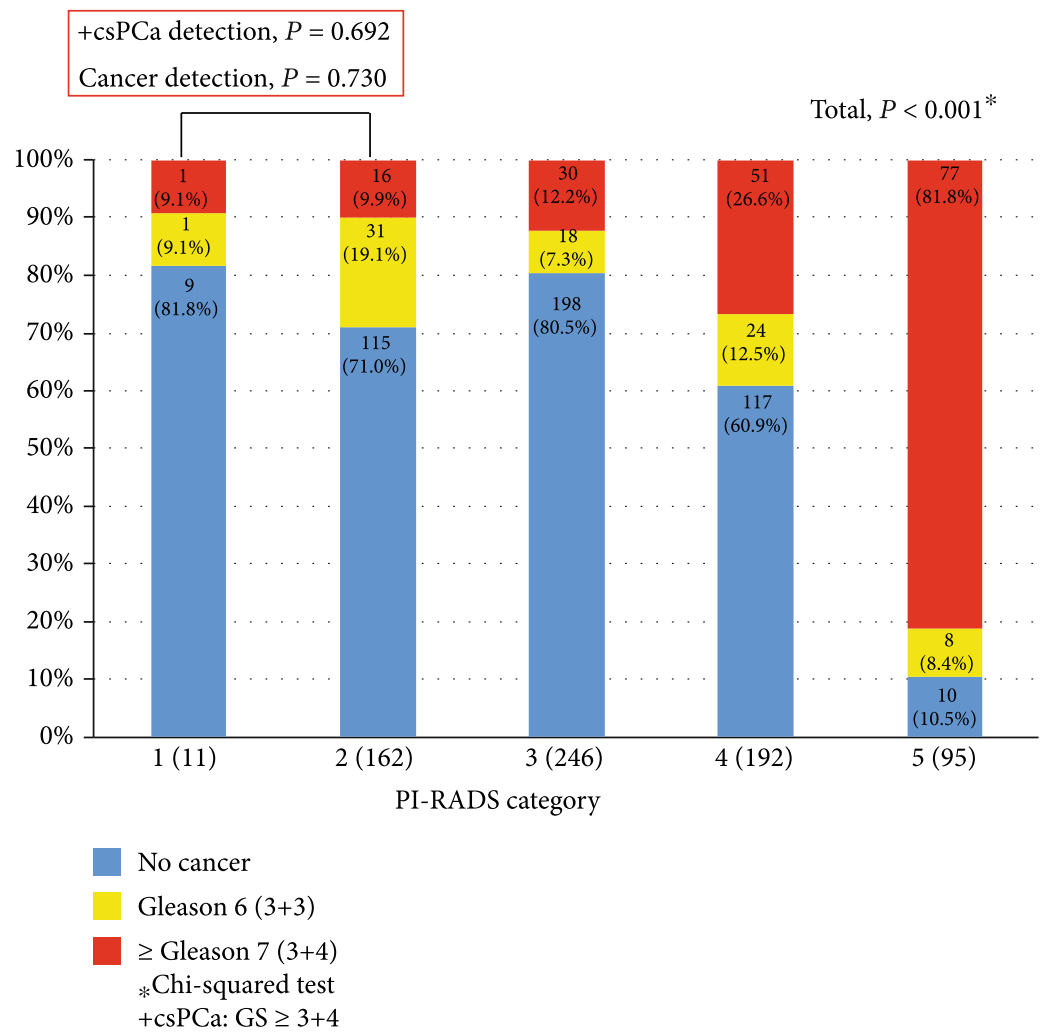

FIGURE 1: Prostate cancer detection stratified by Prostate Imaging-Reporting and Data System version 2 (PI-RADSv2) category in transrectal ultrasound (TRUS)/magnetic resonance imaging (MRI) fusion biopsy cohort.

2.6. Statistical Analyses. Comparative analyses between PI-RADS categorical groups were conducted using a chi-squared test to evaluate the performance difference in predicting (1) TRUS/MRI fusion biopsy and (2) RP pathologic outcomes among different patient cohorts. In the subgroup analysis of PI-RADSv2 category 1 and 2 groups in the RP total cohort, the chi-squared test was used for categorical variables while independent $t$-test or Mann-Whitney $U$ test was used for continuous variables to compare clinicopathological characteristics between the two groups. In addition, we performed receiver operating characteristic (ROC) curve analysis for AP prediction. We also performed logistic regression analyses to evaluate significant variables associated with AP. All statistical analyses were performed using IBM SPSS Statistics ver. 22.0 (Armonk, NY, USA), statistical package for R, ver. 2.13.2 (R Foundation for Statistical Computing (https://www.r-project.org/)). Statistical significance was considered when a two-sided $P$ value was less than 0.05 .

\section{Results}

3.1. TRUS/MRI Fusion Biopsy Outcomes. Pathologic outcomes of TRUS/MRI fusion biopsy stratified by PI-RADSv2 categories are shown in Figure 1. There were significant $(P<0.001)$ differences in $\mathrm{PCa} / \mathrm{cs} \mathrm{PCa}$ detection rates between groups stratified by PI-RADSv2 category. In contrast, there were no significant differences in these rates between PI-RADSv2 category 1 and 2 groups (all $P>0.05$ ). Notably, patients with PI-RADSv2 category 1 or 2 had nonnegligible number of csPCa (9.1\% and $9.9 \%$, respectively).

3.2. RP Pathologic Outcomes in Total Cohort. Among a total of 1403 patients, the rate of AP in RP specimen was significantly correlated with PI-RADS category resulting equal to $33.1 \%$ vs. $41.9 \%$ vs. $56.6 \%$ vs. $86.2 \%$ in the presence of PI-RADS category 1 or 2 vs. 3 vs. 4 vs. 5, respectively $(P<0.001$, Table 1$)$. ROC curve analysis for AP prediction showed an area under the curve (AUC) of 0.747 (95\% confidence interval (CI): 0.721-0.773) for PI-RADS category (Figure 2).

3.3. Comparison of PI-RADS Categories 1 and 2 in the Subgroup of the RP Cohort. Results of comparative analyses of clinicopathological features between groups of PI-RADSv2 categories $1(n=33)$ and $2(n=311)$ are summarized in Table 2 . There were significant differences in PSAD (categorical), prostate volume on TRUS, biopsy GS, and NCCN criteria (all $P<0.05$ ) between the two groups. However, there were no significant differences in pathologic outcomes between the two groups (Table 3). In multivariate logistic regression analyses for evaluating variables associated with AP, diabetes, prebiopsy PSA, total prostate volume, and biopsy GS were found to be significant predictors of AP (all $P<0.05$, Table 4). However, PI-RADSv2 category (1 vs. 2) had no significant association with AP, even in univariate analysis ( $P=0.299$; $95 \% \mathrm{CI}$ : 0.664-3.779). 
TABLE 1: Comparative analyses of pathologic outcomes according to PI-RADSv2 category in radical prostatectomy cohort.

\begin{tabular}{|c|c|c|c|c|c|}
\hline$N(\%)$ & $\begin{array}{l}\text { PI-RADS categories } 1 \text { and } 2 \\
\qquad(N=344)\end{array}$ & $\begin{array}{l}\text { PI-RADS category } 3 \\
\quad(N=167)\end{array}$ & $\begin{array}{l}\text { PI-RADS category } 4 \\
\qquad(N=385)\end{array}$ & $\begin{array}{l}\text { PI-RADS category } 5 \\
\qquad(N=507)\end{array}$ & $P$ \\
\hline Pathologic Gleason score & & & & & $<0.001$ \\
\hline 6 & $30(8.7)$ & $15(9.0)$ & $11(2.9)$ & $1(0.2)$ & \\
\hline $3+4$ & $214(62.2)$ & $92(55.1)$ & $188(48.8)$ & $100(19.7)$ & \\
\hline $4+3$ & $85(24.7)$ & $49(29.3)$ & $152(39.5)$ & $233(46.0)$ & \\
\hline$\geq 8$ & $15(4.4)$ & $11(6.6)$ & $34(8.8)$ & $173(34.1)$ & \\
\hline Pathologic stage & & & & & $<0.001$ \\
\hline$\leq \mathrm{T} 2$ & $305(88.7)$ & $137(82.0)$ & $293(76.1)$ & $217(42.8)$ & \\
\hline$\geq \mathrm{T} 3$ & $39(11.3)$ & $30(18.0)$ & $92(23.9)$ & $290(57.2)$ & \\
\hline Adverse pathology, yes & $114(33.1)$ & $70(41.9)$ & $218(56.6)$ & $437(86.2)$ & $<0.001$ \\
\hline
\end{tabular}

PI-RADSv2: Prostate Imaging-Reporting and Data System version 2.
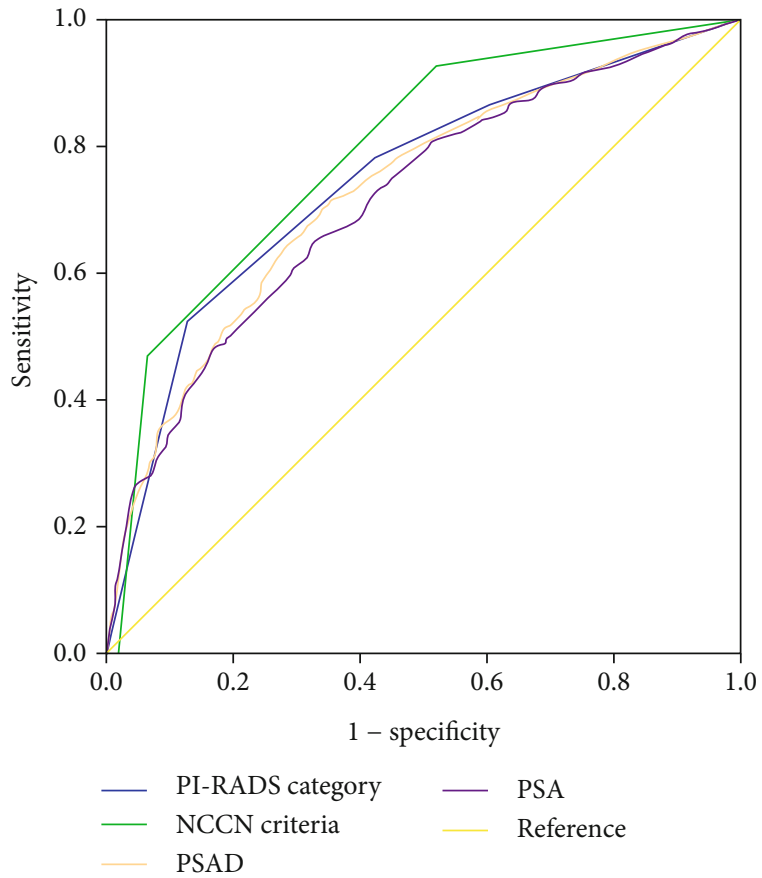

\begin{tabular}{lccc}
\hline & AUC & $95 \%$ CI & $P$ \\
\hline PI-RADS category & 0.747 & $0.721-0.773$ & $<0.001$ \\
NCCN criteria & 0.792 & $0.768-0.816$ & $<0.001$ \\
PSAD & 0.732 & $0.705-0.758$ & $<0.001$ \\
PSA & 0.717 & $0.690-0744$ & $<0.001$ \\
\hline
\end{tabular}

FIgURE 2: Receiver operating characteristic (ROC) curves for adverse pathology prediction in the radical prostatectomy (RP) cohort.

\section{Discussion}

Recently, there has been more emphasis on the role of mpMRI in diagnostic pathway for early PCa after the publication of two landmark studies [7, 8]. The PROMIS trial reported NPV of $89 \%$ for csPCa using 1.5-tesla mpMRI followed by both transrectal ultrasound (TRUS) biopsy and template prostate mapping (TPM) biopsy as a reference [7]. After that, the PRECISION trial compared a conventional diagnostic pathway (12-core TRUS-biopsy) and an MRIdirected pathway (MRI-targeted biopsy without systematic biopsy) and found that the MRI-targeted biopsy group showed superiority to conventional biopsy group in detection rate of both csPCa (38\% vs. $22 \%$; $95 \%$ confidence interval (CI): 4-20; $P=0.005)$ and clinically insignificant PCa (9\% vs. $22 \%$; $95 \%$ CI: -19 to $-7 ; P<0.001)$ [8].

Even with these practice-changing studies, obvious concern for this provision is still on debate. Based on PROMIS data, $11 \%$ of Gleason score (GS) $\geq 4+3$ and $28 \%$ of GS $\geq 3+4$ were missed on mpMRI [7]. They were even missed on TPM biopsy. In the PRECISION trial, $28 \%$ of patients in the MRI-targeted biopsy group did not undergo biopsy because they had a negative result on mpMRI [8]. In those patients, a significant number of csPCa might have been missed. Thus, systematic biopsies are still recommended for these patients although they have higher risk of complications along with economic burdens [9-11]. Several studies have reported a strong correlation between higher grade tumors and higher category of PI-RADS [12, 13, 19, 21]. Lim et al. [21] have demonstrated that higher PI-RADSv2 categories for GS $3+4$ PCa detected at TRUS biopsy are associated with upgrading to GS $4+3$ cancer and the presence of EPE after RP. They also reported that PI-RADSv2 category $\geq 3$ was $100 \%$ sensitive for diagnosing GS $4+3$ tumors. In the current study, we also found these strong correlations both in biopsy and RP pathologic outcomes (Figure 1 and Table 1). The rate of AP in RP specimen was significantly correlated with PI-RADS category: $33.1 \%$ vs. $41.9 \%$ vs. $56.6 \%$ vs. $86.2 \%$ in PI-RADS category 1 or 2 vs. 3 vs. 4 vs. 5, respectively $(P<0.001$, Table 1$)$.

Regarding PI-RADSv2 category 1 or 2 , also known as "negative" mpMRI, several studies have reported a high NPV of mpMRI to exclude csPCa [7, 29, 30]. Subsequently, many authorities have recommended surveillance alone without biopsy for patients with PI-RADSv2 category 1 or 2 [31]. However, there is paucity of data concerning the intermediate and long-term follow-up and their correlation with $\mathrm{RP}$ pathologic outcomes [11]. In addition, according to a 
TABLE 2: Baseline characteristics of PI-RADSv2 category 1 and 2 groups in subgroup of the radical prostatectomy cohort.

\begin{tabular}{|c|c|c|c|}
\hline$N(\%)$ or mean $\pm \mathrm{SD}$ & PI-RADSv2 category $1(N=33)$ & PI-RADSv2 category $2(N=311)$ & $P$ \\
\hline Age & $65.6 \pm 6.5$ & $65.2 \pm 7.3$ & 0.778 \\
\hline BMI & $24.1 \pm 2.4$ & $24.4 \pm 2.7$ & 0.562 \\
\hline Prebiopsy PSA & $6.78 \pm 3.8$ & $8.86 \pm 13.3$ & 0.371 \\
\hline PSAD, continuous & $0.17 \pm 0.95$ & $0.29 \pm 0.64$ & 0.258 \\
\hline PSAD, categorical & & & 0.003 \\
\hline$\leq 0.15$ & $22(66.7)$ & $120(38.6)$ & \\
\hline$>0.15$ & $11(33.3)$ & $191(61.4)$ & \\
\hline Prostate volume & $42.1 \pm 10.7$ & $35.9 \pm 14.4$ & 0.004 \\
\hline Biopsy Gleason score & & & 0.002 \\
\hline 6 & $25(75.8)$ & $135(43.4)$ & \\
\hline $3+4$ & $3(9.1)$ & $100(32.2)$ & \\
\hline $4+3$ & $4(12.1)$ & $53(17.0)$ & \\
\hline$\geq 8$ & $1(3.0)$ & $23(7.4)$ & \\
\hline NCCN criteria & & & 0.002 \\
\hline Low & $22(66.7)$ & $112(36.0)$ & \\
\hline Intermediate & $9(27.3)$ & $164(52.7)$ & \\
\hline High & $1(3.0)$ & $35(11.3)$ & \\
\hline
\end{tabular}

BMI: body mass index; NCCN: National Comprehensive Cancer Network; PI-RADSv2: Prostate Imaging-Reporting and Data System version 2; PSA: prostatespecific antigen; PSAD: PSA density; SD: standard deviation.

TABLE 3: Comparative analysis results of pathologic outcomes between PI-RADSv2 category 1 and 2 groups in the radical prostatectomy cohort.

\begin{tabular}{lccc}
\hline & $\begin{array}{c}\text { PI-RADSv2 } \\
\text { category 1 }(N=33)\end{array}$ & $\begin{array}{c}\text { PI-RADSv2 category } \\
2(N=311)\end{array}$ & $P$ \\
\hline $\begin{array}{l}\text { Pathologic GS } \\
\leq 3+4\end{array}$ & $26(78.8 \%)$ & $218(70.1 \%)$ & 0.420 \\
$\quad \geq 4+3$ & $7(21.2 \%)$ & $93(29.9 \%)$ & \\
$\begin{array}{l}\text { Pathologic } \\
\text { stage }\end{array}$ & & & 1.000 \\
$\quad \leq \mathrm{T} 2$ & $30(90.0 \%)$ & $275(88.4 \%)$ & \\
$\quad \geq \mathrm{T} 3$ & $3(9.1 \%)$ & $36(11.6 \%)$ & \\
$\begin{array}{l}\text { Adverse } \\
\text { pathology, yes }\end{array}$ & $9(27.3 \%)$ & $105(33.8 \%)$ & 0.561 \\
\hline
\end{tabular}

GS: Gleason score; PI-RADSv2: Prostate Imaging-Reporting and Data System version 2.

recent meta-analysis, there is no definitive conclusion about the NPV of mpMRI [4]. Voss et al. [10] have shown that over $28.9 \%$ of upgraded cases would have been missed, including GS $\geq 4+3$ tumors. Even with the combination of PI-RADS category $\geq 3$ and/or PSA density of $\geq 0.15$, a significant number of men with intermediate-risk disease were misclassified. In the current study, we found that patients even with PI-RADSv2 categories 1 and 2 had a significant number of csPCa after TRUS/MRI fusion biopsy (17/173, 9.8\% (Figure 1)). Among the patients who underwent RP with PI-RADS categories 1 and 2 in preoperative mpMRI, the rate of AP in RP specimen was as high as $33.1 \%$ (Table 1 ). With these findings, we can extrapolate to conclude that a significant number of patients with negative mpMRI may harbor csPCa and experience GS upgrading in their final pathology.

Current data on negative mpMRI are still lacking and studies regarding PI-RADS category 1 are scarce $[9,11-14$, 20]. Bianchi et al. [20] have demonstrated the correlation between higher PI-RADS category and high probability of upgrading and upstaging after comparing PI-RADS category $2 / 3$ vs. 4 vs. 5 and PI-RADS $2 / 3$ versus $4 / 5$. However, they did not include category 1 in their study. Until now, it remains unclear whether we can safely obviate biopsy, especially for PI-RADSv2 category 1 lesion. Notably, PI-RADSv2 categories 1 and 2 are not so different from each other in assessment. Category 1 lesion is defined as homogenous or normal signal intensity while category 2 lesion is defined as "indistinct" hypointense lesion on T2WI or ADC [3]. It would be essential to evaluate the performance of PI-RADS category 1 in predicting pathologic outcomes for further implementing surveillance strategies in this group of patients. In the current study, we found that there was no significant difference in $\mathrm{PCa} / \mathrm{csPCa}$ detection rate between PI-RADSv2 category 1 and 2 groups after TRUS/MRI fusion biopsy (Figure 1). In the RP cohort, there were no significant differences in the rate of AP between the two groups (27.3\% vs. $33.8 \%, P=0.561$, Table 3 ). Consequently, these results emphasize that performance improvement of mpMRI is needed for lesions classified as PI-RADSv2 categories 1 and 2. In addition, PI-RADSv2 category 1 should not be overlooked in the current mpMRI era.

In order to improve the performance of mpMRI in these groups, risk-assessment tools such as PSA, PSAD, Prostate Health Index, four kallikrein (4K) score, and Prostate Cancer gene 3 (PCA3) need to be combined [32-34]. In a recent 
TABLE 4: Univariate and multivariate logistic regression analyses to detect adverse pathology in patients of PI-RADSv2 category 1 and 2 groups.

\begin{tabular}{lccccc}
\hline Variables & OR & Univariate & & \multicolumn{2}{c}{ Multivariate } \\
$95 \%$ CI & OR & $0.986-1.066$ \\
\hline Age & 1.034 & $1.001-1.068$ & 0.040 & 1.025 & 0.204 \\
BMI & 0.924 & $0.846-1.009$ & 0.080 & 0.907 & $0.820-1.003$ \\
DM & 2.204 & $1.206-4.027$ & 0.010 & 2.639 & $1.281-5.436$ \\
HTN & 1.052 & $0.670-1.654$ & 0.825 & & 0.0087 \\
Prebiopsy PSA & 1.044 & $1.010-1.078$ & 0.010 & 1.050 & $0.006-1.095$ \\
Prostate volume & 0.973 & $0.955-0.991$ & 0.004 & 0.976 & $0.956-0.997$ \\
Biopsy Gleason score & 7.240 & $4.377-11.977$ & $<0.001$ & 6.438 & $3.823-10.842$ \\
NCCN criteria & 4.836 & $3.119-7.501$ & $<0.001$ & 0.603 & $0.167-2.177$ \\
Clinical stage & 1.329 & $0.890-1.985$ & 0.165 & & 0.025 \\
PI-RADSv2, 1 vs. 2 & 1.585 & $0.664-3.779$ & 0.299 & & 0.001 \\
\hline
\end{tabular}

BMI: body mass index; DM: diabetes mellitus; HTN: hypertension; NCCN: National Comprehensive Cancer Network; OR: odds ratio; PI-RADSv2: Prostate Imaging-Reporting and Data System version 2; PSA: prostate-specific antigen.

prospective observational study, Lopci et al. [32] have reported that the use of ${ }^{68} \mathrm{Ga}$-labeled prostate-specific membrane antigen positron emission tomography/computerized tomography has significant performance in predicting csPCa among negative mpMRI patients.

The current study has some limitations. First, even with a large tertiary institution study, the retrospective study design was a crucial drawback. In addition, our study was based on a heterogeneous study cohort. For example, RP was conducted by several surgeons with several modalities and biopsy was conducted without standardized inclusion criteria. Finally, we did not conduct a rereview of pathologic slides. Accordingly, subsequent misclassification of some lesions might have affected outcomes. Further larger studies are warranted to validate and generalize our results.

\section{Conclusions}

The present study revealed that there were no significant differences between PI-RADSv2 category 1 and 2 groups in terms of the detection rate of csPCa. In addition, these groups had similar performance to predict pathologic outcomes at RP. Consequently, these two categories may be unified into a single category. However, these two categories defined as negative mpMRI do not guarantee the absence of AP, as with csPCa. Performance improvement of mpMRI is needed for these lesions. In addition, PI-RADSv2 category 1 should not be overlooked in the current mpMRI era.

\section{Data Availability}

The data that support the findings of this study are available from the corresponding author upon reasonable request.

\section{Conflicts of Interest}

All the authors had no conflicts of interest in this study.

\section{Authors' Contributions}

Jung Kwon Kim and Sung Kyu Hong contributed to manuscript conception. Jung Kwon Kim contributed to manuscript preparation. Jung Kwon Kim and Sung Il Hwang contributed to statistical analysis. Hak Jong Lee, Gheeyoung Choe, and Sung Kyu Hong contributed to the internal review of the draft. All authors approved the manuscript.

\section{References}

[1] N. Mottet, J. Bellmunt, M. Bolla et al., "EAU-ESTRO-SIOG guidelines on prostate cancer. Part 1: screening, diagnosis, and local treatment with curative intent," European Urology, vol. 71, no. 4, pp. 618-629, 2017.

[2] J. O. Barentsz, J. Richenberg, R. Clements et al., "ESUR prostate MR guidelines 2012," European Radiology, vol. 22, no. 4, pp. 746-757, 2012.

[3] J. C. Weinreb, J. O. Barentsz, P. L. Choyke et al., "PI-RADS prostate imaging-reporting and data system: 2015, version 2," European Urology, vol. 69, no. 1, pp. 16-40, 2016.

[4] S. Woo, C. H. Suh, S. Y. Kim, J. Y. Cho, and S. H. Kim, “Diagnostic performance of prostate imaging reporting and data system version 2 for detection of prostate cancer: a systematic review and diagnostic meta-analysis," European Urology, vol. 72, no. 2, pp. 177-188, 2017.

[5] J. J. Fütterer, A. Briganti, P. de Visschere et al., "Can clinically significant prostate cancer be detected with multiparametric magnetic resonance imaging? A systematic review of the literature," European Urology, vol. 68, no. 6, pp. 1045-1053, 2015.

[6] J. Barentsz, M. de Rooij, G. Villeirs, and J. Weinreb, "Prostate imaging-reporting and data system version 2 and the implementation of high-quality prostate magnetic resonance imaging," European Urology, vol. 72, no. 2, pp. 189-191, 2017.

[7] H. U. Ahmed, A. el-Shater Bosaily, L. C. Brown et al., "Diagnostic accuracy of multi-parametric MRI and TRUS biopsy in prostate cancer (PROMIS): a paired validating confirmatory study," The Lancet, vol. 389, no. 10071, pp. 815-822, 2017.

[8] V. Kasivisvanathan, A. S. Rannikko, M. Borghi et al., "MRItargeted or standard biopsy for prostate-cancer diagnosis," 
The New England Journal of Medicine, vol. 378, no. 19, pp. 1767-1777, 2018.

[9] N. Branger, T. Maubon, M. Traumann et al., "Is negative multiparametric magnetic resonance imaging really able to exclude significant prostate cancer? The real-life experience," BJU International, vol. 119, no. 3, pp. 449-455, 2017.

[10] J. Voss, R. Pal, S. Ahmed, M. Hannah, A. Jaulim, and T. Walton, "Utility of early transperineal template-guided prostate biopsy for risk stratification in men undergoing active surveillance for prostate cancer," BJU International, vol. 121, no. 6, pp. 863-870, 2018.

[11] V. Panebianco, G. Barchetti, G. Simone et al., "Negative Multiparametric Magnetic Resonance Imaging for Prostate Cancer: What's Next?," European Urology, vol. 74, no. 1, pp. 48-54, 2018.

[12] H. Cash, A. Maxeiner, C. Stephan et al., "The detection of significant prostate cancer is correlated with the prostate imaging reporting and data system (PI-RADS) in MRI/transrectal ultrasound fusion biopsy," World Journal of Urology, vol. 34, no. 4, pp. 525-532, 2016.

[13] A. Borkowetz, I. Platzek, M. Toma et al., "Evaluation of prostate imaging reporting and data system classification in the prediction of tumor aggressiveness in targeted magnetic resonance imaging/ultrasound-fusion biopsy," Urologia Internationalis, vol. 99, no. 2, pp. 177-185, 2017.

[14] J. Y. An, A. Sidana, S. A. Holzman et al., "Ruling out clinically significant prostate cancer with negative multi-parametric MRI," International Urology and Nephrology, vol. 50, no. 1, pp. 7-12, 2018.

[15] S. L. Hofbauer, A. Maxeiner, B. Kittner et al., "Validation of prostate imaging reporting and data system version 2 for the detection of prostate cancer," The Journal of Urology, vol. 200, no. 4, pp. 767-773, 2018.

[16] J. K. Mullins, D. Bonekamp, P. Landis et al., "Multiparametric magnetic resonance imaging findings in men with low-risk prostate cancer followed using active surveillance," BJU International, vol. 111, no. 7, pp. 1037-1045, 2013.

[17] B. Helpap and L. Egevad, "Modified Gleason grading. An updated review," Histology and Histopathology, vol. 24, no. 5, pp. 661-666, 2009.

[18] A. Borkowetz, I. Platzek, M. Toma et al., "Direct comparison of multiparametric magnetic resonance imaging (MRI) results with final histopathology in patients with proven prostate cancer in MRI/ultrasonography-fusion biopsy," BJU International, vol. 118, no. 2, pp. 213-220, 2016.

[19] O. de Cobelli, D. Terracciano, E. Tagliabue et al., "Predicting pathological features at radical prostatectomy in patients with prostate cancer eligible for active surveillance by multiparametric magnetic resonance imaging," PLoS One, vol. 10, no. 10, article e0139696, 2015.

[20] R. Bianchi, G. Cozzi, G. Petralia et al., "Multiparametric magnetic resonance imaging and frozen-section analysis efficiently predict upgrading, upstaging, and extraprostatic extension in patients undergoing nerve-sparing robotic-assisted radical prostatectomy," Medicine, vol. 95, no. 40, article e4519, 2016.

[21] C. S. Lim, M. D. F. McInnes, T. A. Flood et al., "Prostate imaging reporting and data system, version 2 , assessment categories and pathologic outcomes in patients with Gleason score $3+4$ $=7$ prostate cancer diagnosed at biopsy," AJR. American Journal of Roentgenology, vol. 208, no. 5, pp. 1037-1044, 2017.
[22] C. W. Jeong, Y. H. Park, S. I. Hwang et al., "The role of 3-tesla diffusion-weighted magnetic resonance imaging in selecting prostate cancer patients for active surveillance," Prostate International, vol. 2, no. 4, pp. 169-175, 2014.

[23] H. Lee, S. I. Hwang, H. J. Lee, S. S. Byun, S. E. Lee, and S. K. Hong, "Diagnostic performance of diffusion-weighted imaging for prostate cancer: peripheral zone versus transition zone," PLoS One, vol. 13, no. 6, article e0199636, 2018.

[24] S. I. Hwang, H. J. Lee, S. E. Lee et al., "Value of MR-US fusion in guidance of repeated prostate biopsy in men with PSA $<10$ ng/mL," Clinical Imaging, vol. 53, pp. 1-5, 2019.

[25] S. Lee, S. J. Jeong, S. I. Hwang et al., "Clinical value of core length in contemporary multicore prostate biopsy," PLoS One, vol. 10, no. 4, article e0123704, 2015.

[26] C. Jeldres, N. Suardi, J. Walz et al., "Validation of the contemporary epstein criteria for insignificant prostate cancer in European men," European Urology, vol. 54, no. 6, pp. 13061313, 2008.

[27] M. C. Benson, I. S. Whang, A. Pantuck et al., "Prostate specific antigen density: a means of distinguishing benign prostatic hypertrophy and prostate cancer," The Journal of Urology, vol. 147, 3, Part 2, pp. 815-816, 1992.

[28] J. Mohler, R. R. Bahnson, B. Boston et al., "NCCN clinical practice guidelines in oncology: prostate cancer," Journal of the National Comprehensive Cancer Network, vol. 8, no. 2, pp. 162-200, 2010.

[29] J. Haffner, L. Lemaitre, P. Puech et al., "Role of magnetic resonance imaging before initial biopsy: comparison of magnetic resonance imaging-targeted and systematic biopsy for significant prostate cancer detection," BJU International, vol. 108, no. 8b, pp. E171-E178, 2011.

[30] J. S. Wysock, N. Mendhiratta, F. Zattoni et al., "Predictive value of negative $3 \mathrm{~T}$ multiparametric magnetic resonance imaging of the prostate on 12-core biopsy results," BJU International, vol. 118, no. 4, pp. 515-520, 2016.

[31] National Institute for Health and Care Excellence, "Prostate cancer: diagnosis and management. Clinical guideline [CG175]," September 2019, https://www.nice.org.uk/ guidance/cg175/chapter/1-Recommendations-informationand-decision-support-for-men-with-prostate-cancertheirpartners-ssand-carers-2.

[32] E. Lopci, A. Saita, M. Lazzeri et al., " ${ }^{68}$ Ga-PSMA positron emission tomography/computerized tomography for primary diagnosis of prostate cancer in men with contraindications to or negative multiparametric magnetic resonance imaging: a prospective observational study," The Journal of Urology, vol. 200, no. 1, pp. 95-103, 2018.

[33] A. Friedl, K. Stangl, W. Bauer et al., "Prostate-specific antigen parameters and prostate health index enhance prostate cancer prediction with the in-bore 3-T magnetic resonance imaging-guided transrectal targeted prostate biopsy after negative 12-core biopsy," Urology, vol. 110, pp. 148-153, 2017.

[34] P. J. van Leeuwen, A. Hayen, J. E. Thompson et al., "A multiparametric magnetic resonance imaging-based risk model to determine the risk of significant prostate cancer prior to biopsy," BJU International, vol. 120, no. 6, pp. 774-781, 2017. 